\title{
Differential ceRNA Expression and Interaction Analysis in Coronary Artery Disease
}

\author{
Sheng Kang ${ }^{1}$, Yong $\mathrm{Ye}^{1}$, and Guang Xia ${ }^{1}$ \\ ${ }^{1}$ Shanghai East Hospital
}

May 5, 2020

\begin{abstract}
Background: Previous studies had shown that mRNA, miRNA and lncRNA were associated with cardiovascular diseases. The study was aimed to explore the differential expressions of mRNA, lncRNA and miRNA between coronary artery disease (CAD)and healthy control, and their interaction in CAD. Methods: We investigated the differential expression of ceRNA between CAD and healthy control through data collected from Gene Expression Omnibus (GEO) microarrays. Furthermore, we investigated the biological function of these differential expressions of ceRNAs by Gene Ontology (GO), Kyoto Encyclopedia of Genes and Genomes (KEGG) analyses. Protein-protein interaction (PPI) network was created to identify the hub genes. Biosystems and literature search were performed for signaling pathways and their function of the included differential expression ceRNAs. Results: A total of 456 miRNA expression profiles, 16,325 mRNA expression profiles, and 2,869 lncRNA expression profiles were obtained. Eleven Go and KEGG pathways (count [?]9), top 15 of PPI network node connectivity rank, and top 15 of ceRNA network node degree centrality rank were achieved at the statistical significance level $(\mathrm{P}<0.05)$. We further identified that several differential expressions of ceRNAs and their signaling pathways were associated with CAD through biosystems and literature search. Conclusions: Based on eleven Go and KEGG pathways, top 15 of PPI network node connectivity rank, and top 15 of ceRNA network node degree centrality rank in CAD population, our findings would contribute to further exploration for the molecular mechanism of CAD.
\end{abstract}

\section{Hosted file}

Title Page.doc available at https://authorea.com/users/300453/articles/430110-differentialcerna-expression-and-interaction-analysis-in-coronary-artery-disease 


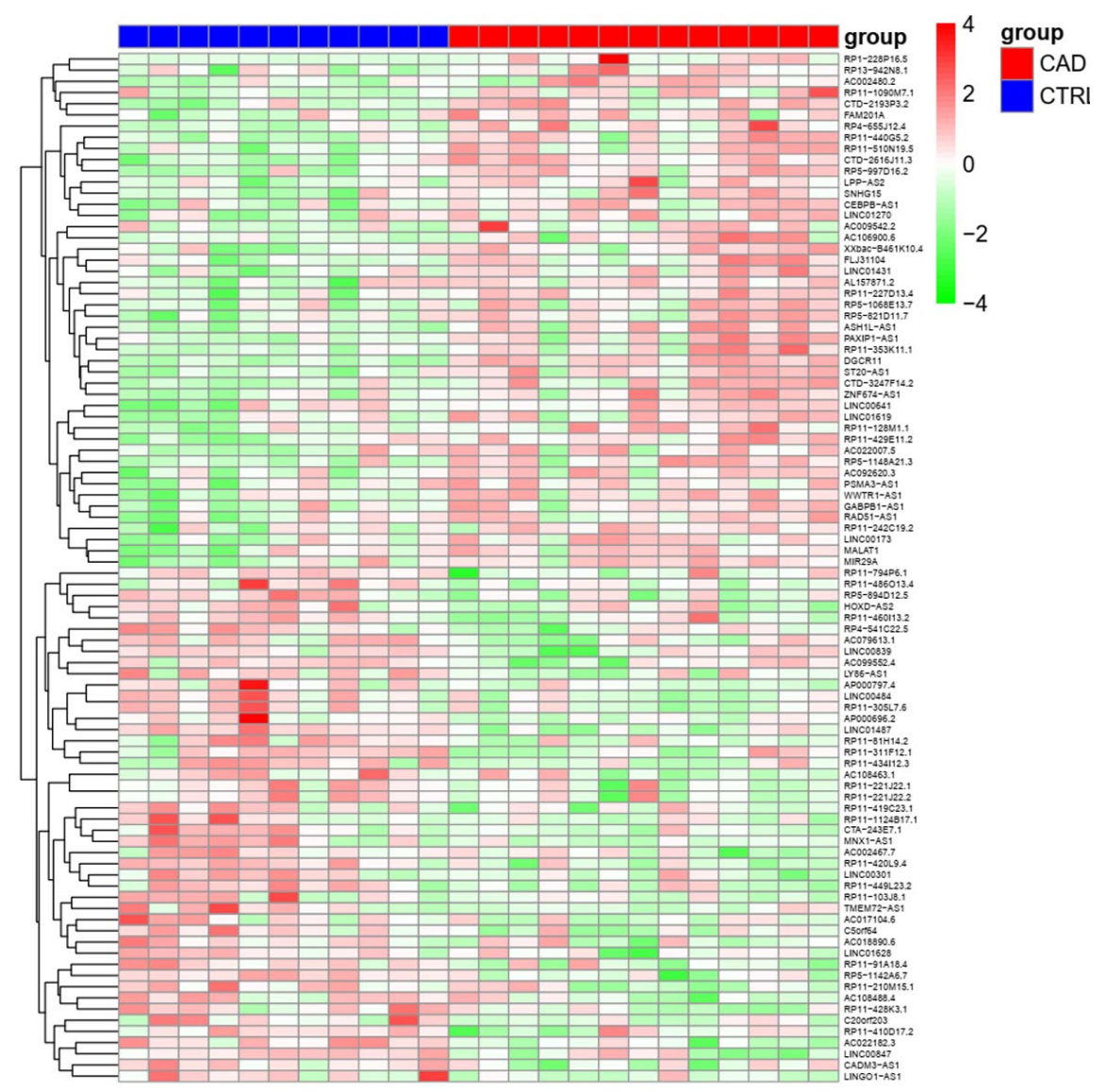




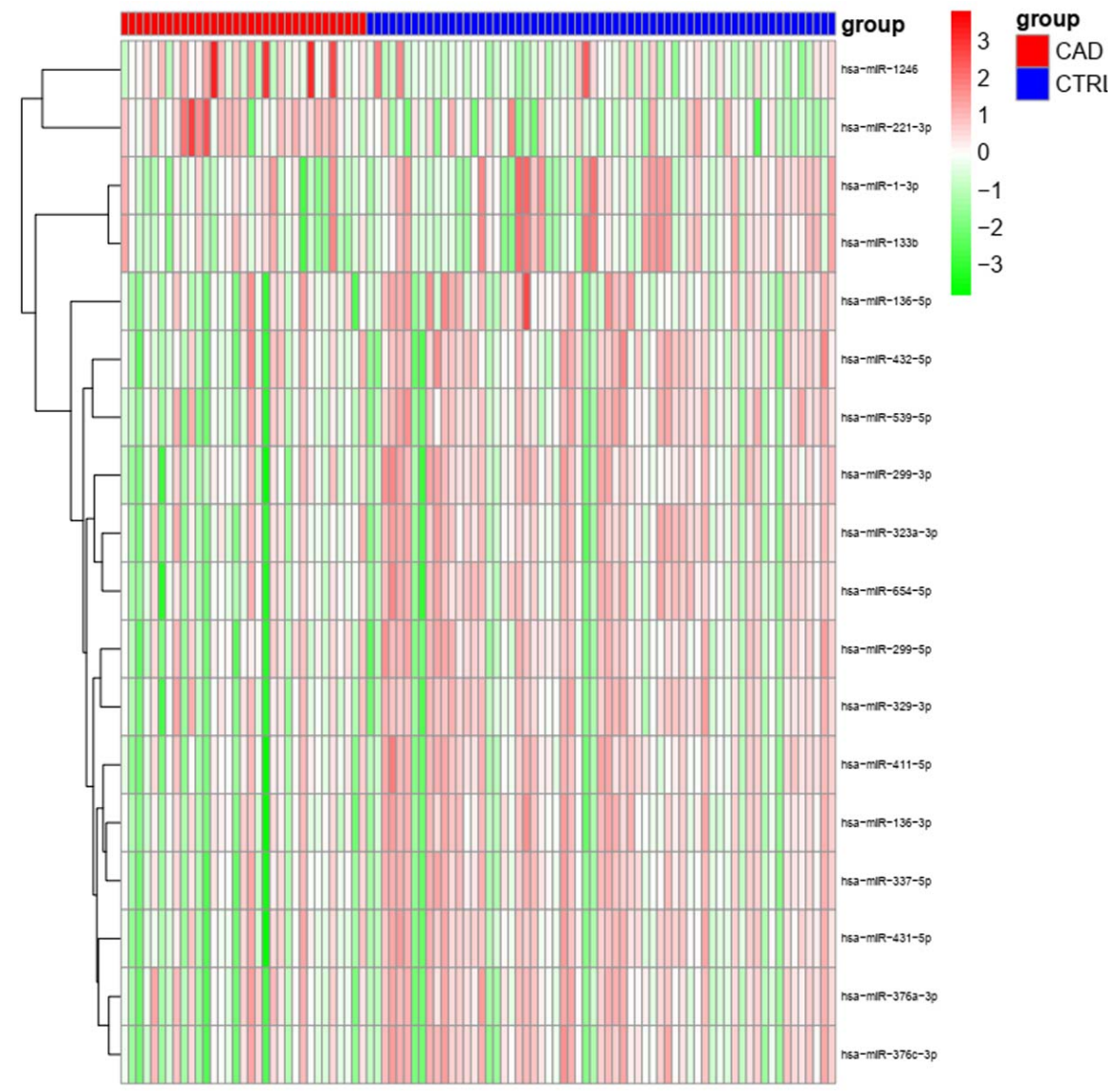




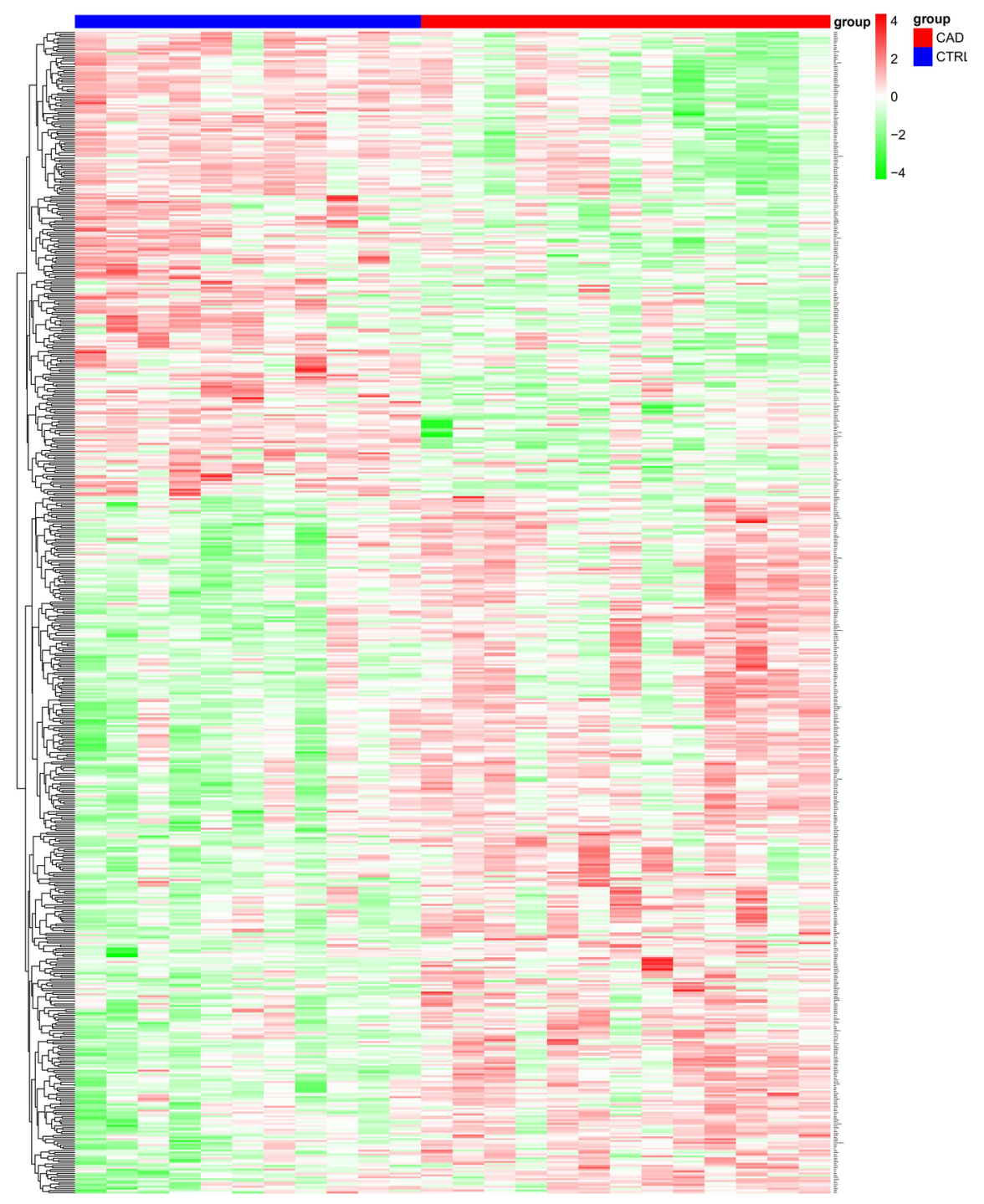




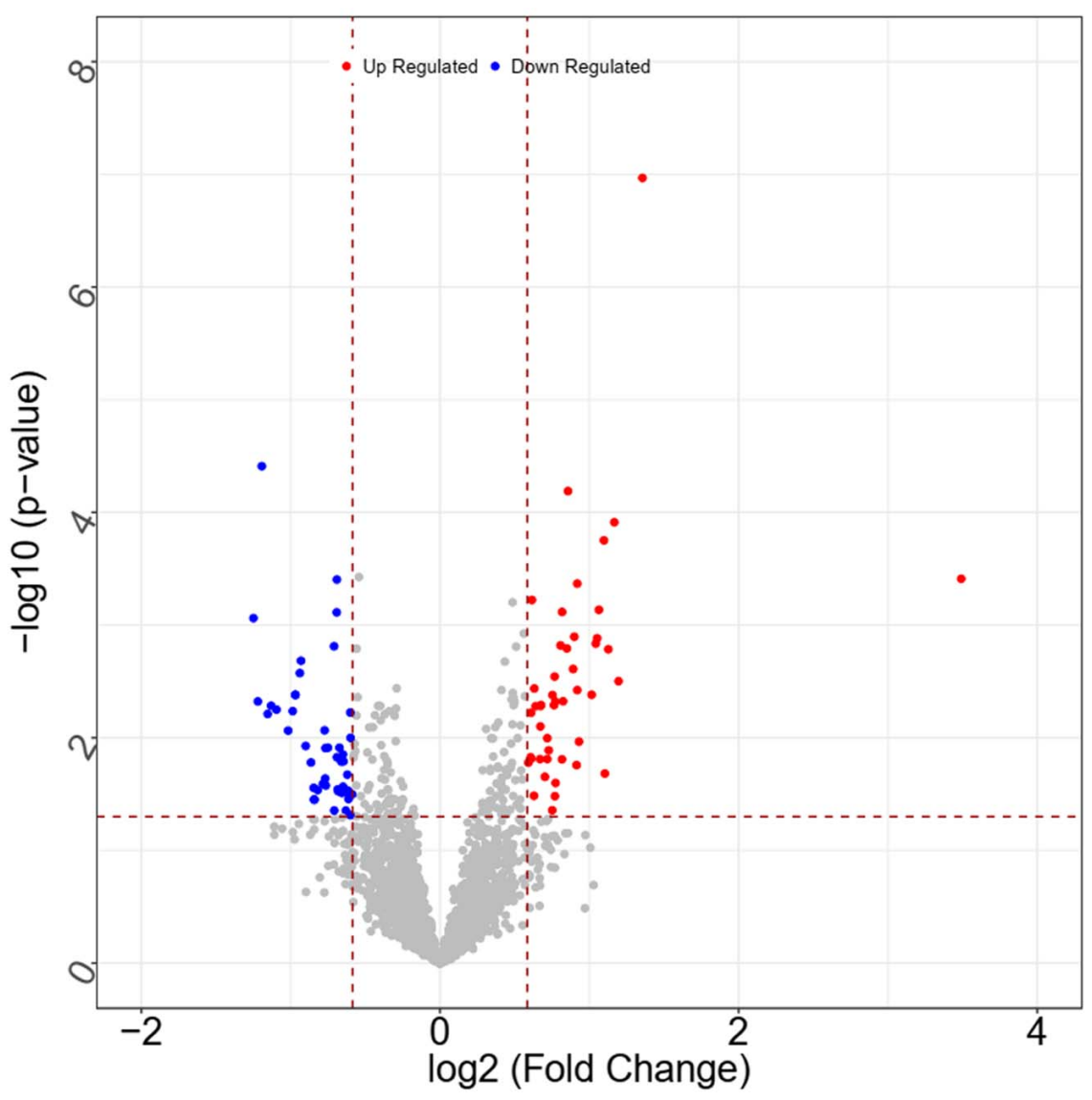




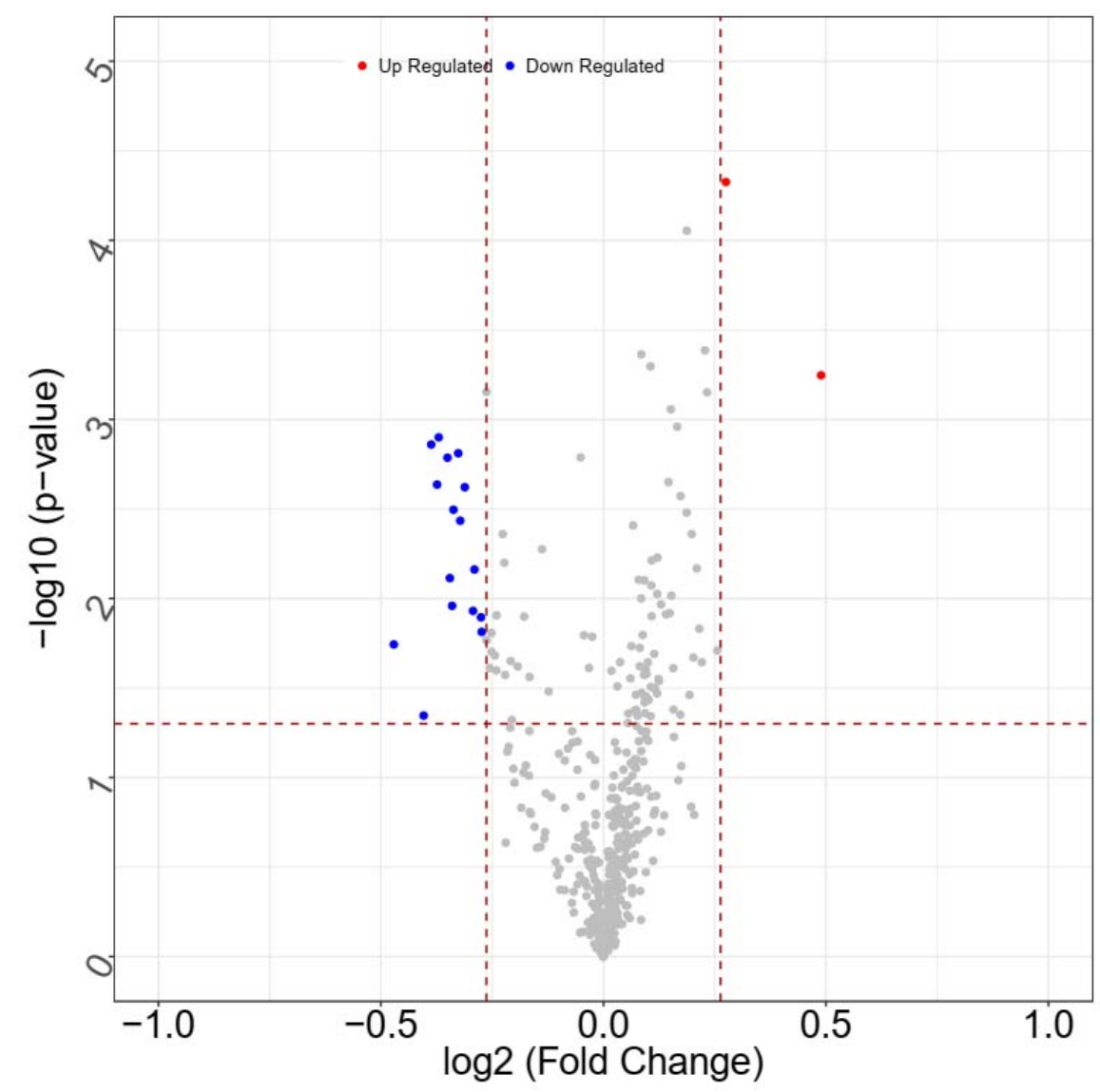



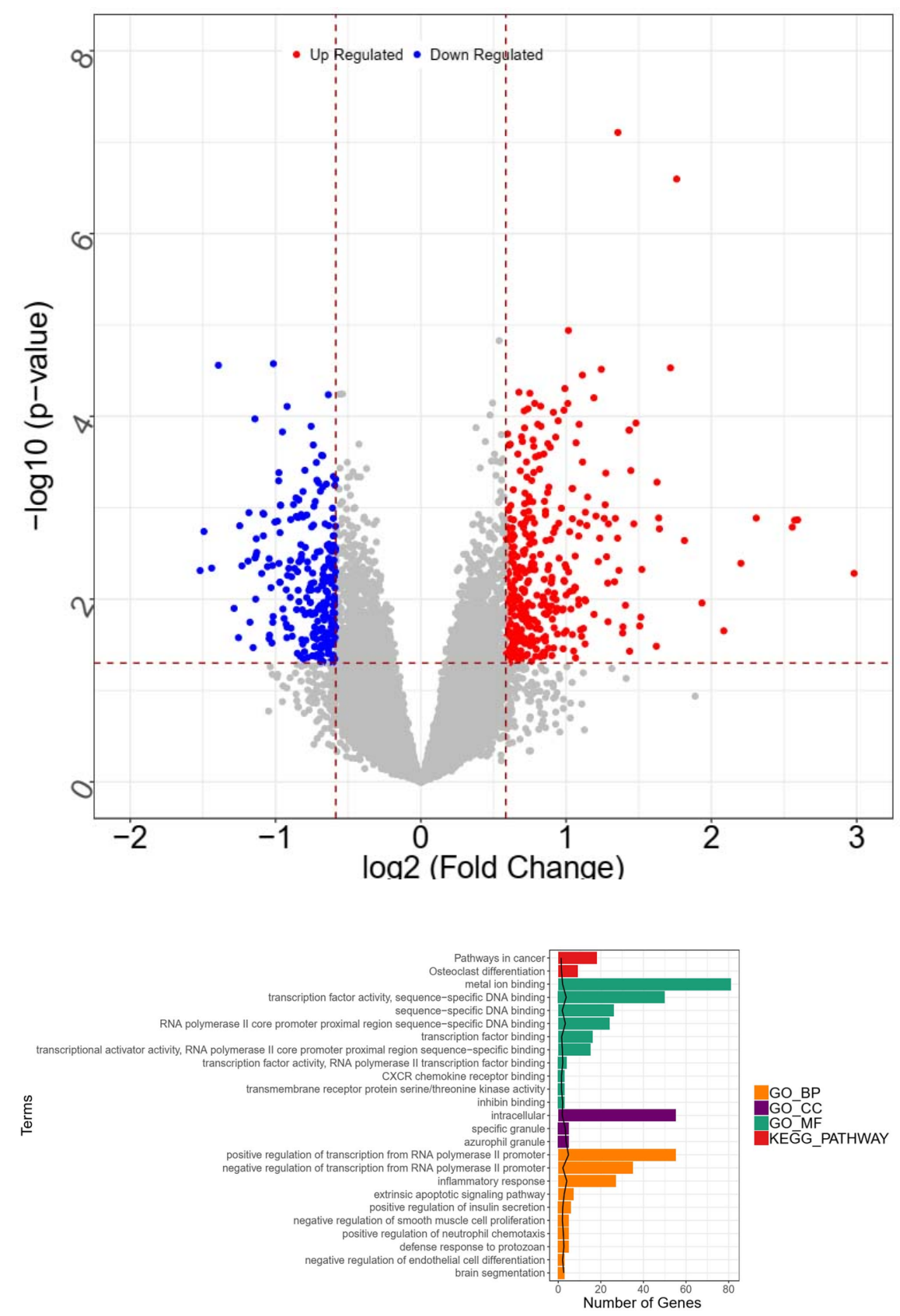

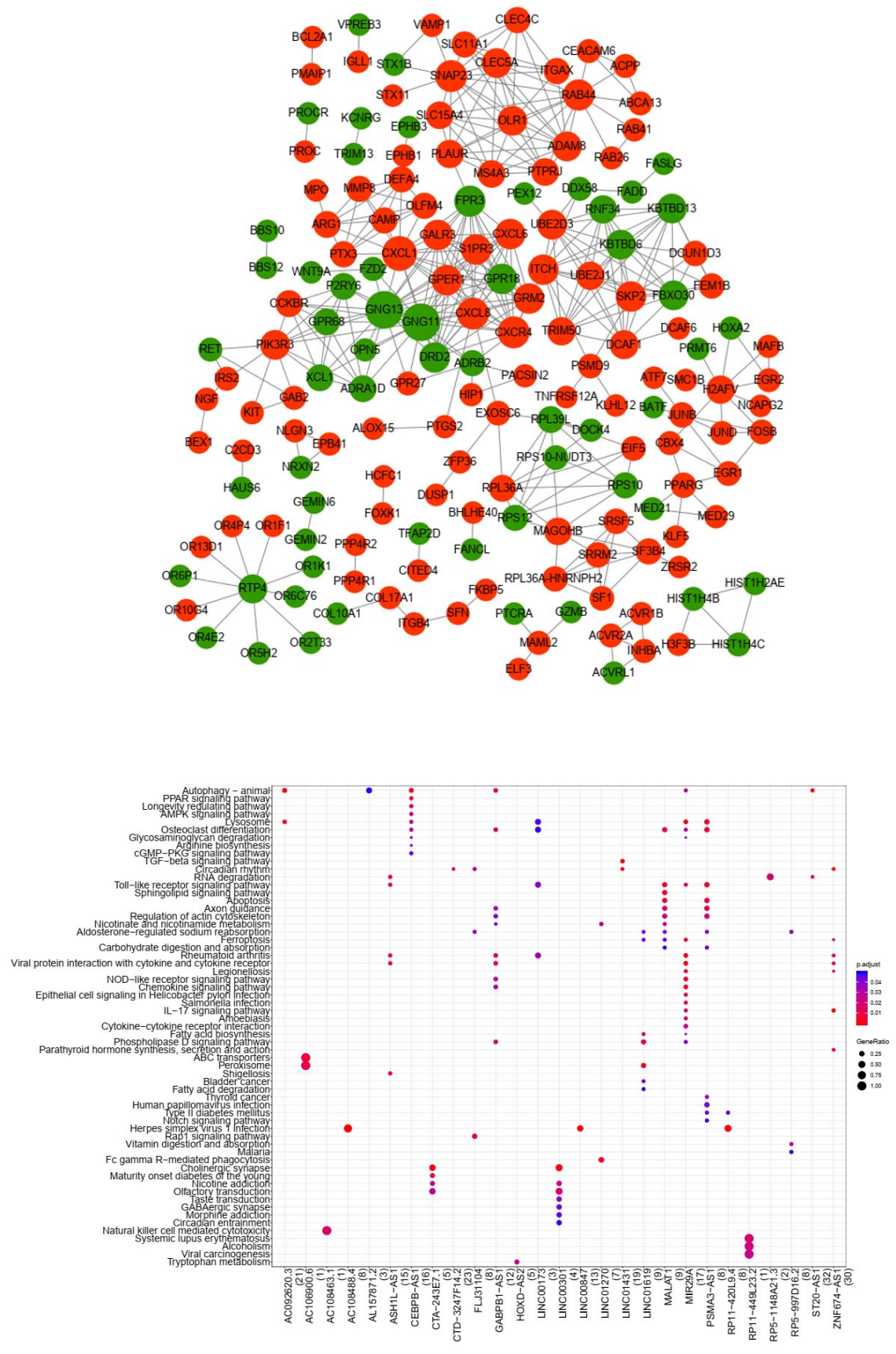

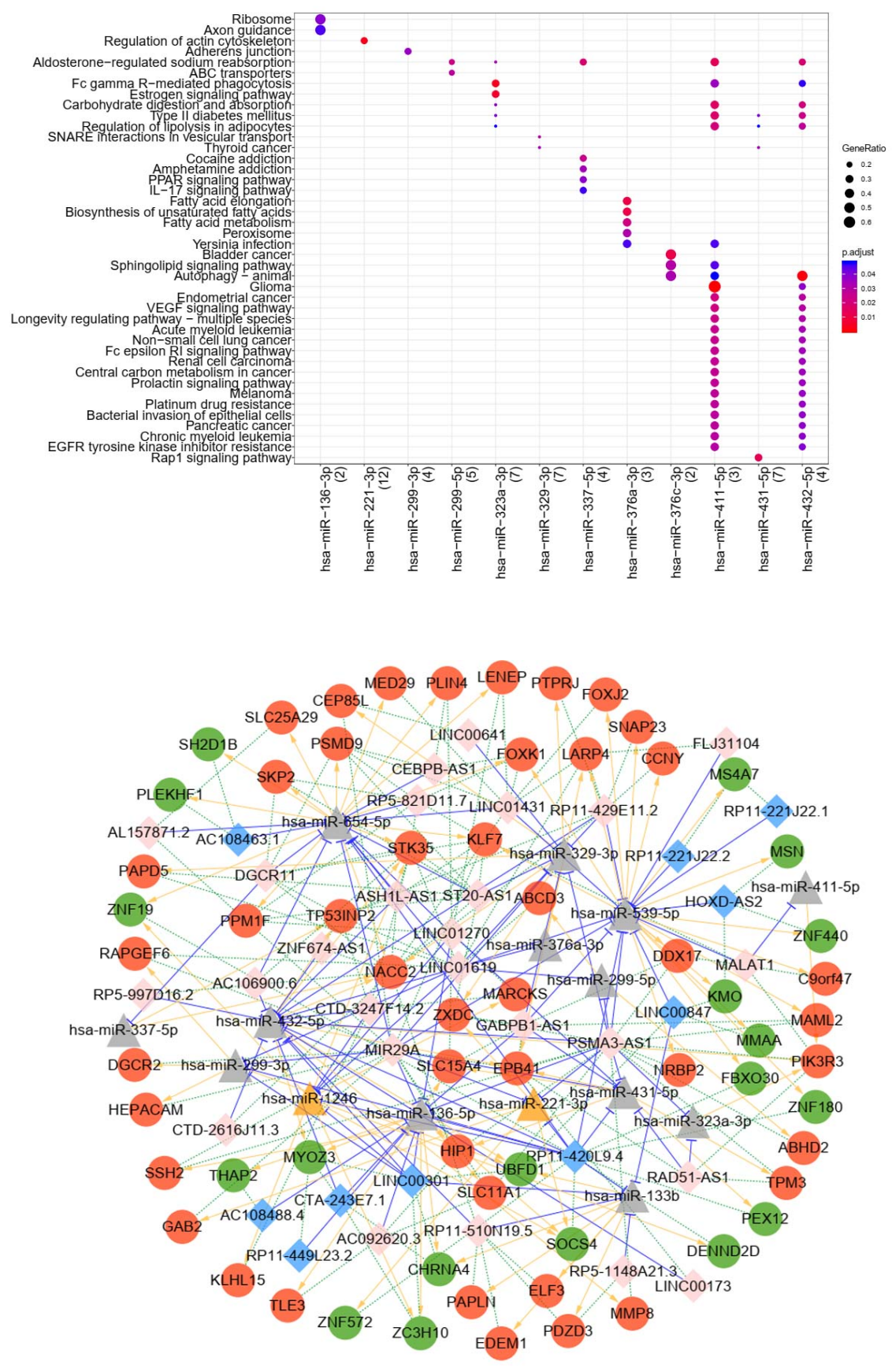